\title{
A Simple Way to Prove the Characterization of Differentiable Quasiconvex Functions
}

\section{Giorgio Giorgi}

Faculty of Economics, University of Pavia, Pavia, Italy

Email: ggiorgi@eco.unipv.it

Received 23 January 2014; revised 23 February 2014; accepted 2 March 2014

Copyright (C) 2014 by author and Scientific Research Publishing Inc.

This work is licensed under the Creative Commons Attribution International License (CC BY). http://creativecommons.org/licenses/by/4.0/

(c) (i) Open Access

\section{Abstract}

We give a short and easy proof of the characterization of differentiable quasiconvex functions.

\section{Keywords}

\section{Quasiconvex Functions, Generalized Convexity}

\section{Introduction}

Quasiconvex functions play an important role in several branches of applied mathematics (e.g. mathematical programming, minimax theory, games theory, etc.) and of economic analysis (production theory, utility theory, etc.). De Finetti [1] was one of the first mathematicians to define quasiconvex functions as those functions $f: X \rightarrow \mathbb{R}, X \subset \mathbb{R}^{n}$ being a convex set, having convex lower level sets, i.e. the set

$$
L(f, \alpha)=\{x: x \in X, f(x) \leq \alpha\}
$$

is convex for every $\alpha \in \mathbb{R}$.

De Finetti did not name this class of functions: the term "quasiconvex (quasiconcave) function" was given subsequently by Fenchel [2]. It is well-known that the above characterization is equivalent to

$$
x^{1}, x^{2} \in X, f\left(x^{2}\right) \leq f\left(x^{1}\right) \Rightarrow f\left(\lambda x^{1}+(1-\lambda) x^{2}\right) \leq f\left(x^{1}\right), \forall \lambda \in[0,1],
$$

i.e., in a more symmetric way,

$$
f\left(\lambda x^{1}+(1-\lambda) x^{2}\right) \leq \max \left\{f\left(x^{1}\right), f\left(x^{2}\right)\right\}, \forall \lambda \in[0,1], \forall x^{1}, x^{2} \in X
$$

When $f$ is differentiable on the open convex set $X \subset \mathbb{R}^{n}$, we have the following characterization of a quasiconvex function. 
Theorem 1. Let $f: X \rightarrow \mathbb{R}$ be differentiable on the open convex set $X \subset \mathbb{R}^{n}$. Then $f$ is quasiconvex on $X$ if and only if

$$
x^{1}, x^{2} \in X, f\left(x^{2}\right) \leq f\left(x^{1}\right) \Rightarrow \nabla f\left(x^{1}\right)\left(x^{2}-x^{1}\right) \leq 0 .
$$

Theorem 1 was given by Arrow and Enthoven [3]; however, these authors prove, in a short and easy way, only the necessary part of the theorem, but not the converse property, whose proof is indeed presented in a quite intricate way by several authors (see, e.g. [4]-[11]). Here we present an easy proof of Theorem 1, by exploiting some results on quasiconvexity of functions of one variable, results therefore suitable for geometrical illustrations. We need two lemmas.

Lemma 1. Let $f: X \rightarrow \mathbb{R}, X \subset \mathbb{R}^{n}$ a convex set. Then $f$ is quasiconvex on $X$ if and only if the restriction of $f$ on each line segment contained in $X$ is a quasiconvex function, i.e. if and only if the function $\theta(t)=f(x+t y)$ is quasiconvex on the interval

$$
I_{x, y}=\{t \in \mathbb{R}: x+t y \in X\} .
$$

Proof. The quasiconvexity of $\theta(t)$ is equivalent to the implication

$$
t_{1}, t_{2} \in I_{x, y}, \theta\left(t_{2}\right) \leq \theta\left(t_{1}\right) \Rightarrow \theta\left(t_{1}+\lambda\left(t_{2}-t_{1}\right)\right) \leq \theta\left(t_{1}\right), \forall \lambda \in[0,1]
$$

By setting $x^{1}=x+t_{1} y, \quad x^{2}=x+t_{2} y$, we have $x^{1}+\lambda\left(x^{2}-x^{1}\right)=x+t_{1} y+\lambda\left(t_{2}-t_{1}\right) y$. The thesis follows by noting that $\theta\left(t_{2}\right) \leq \theta\left(t_{1}\right)$ and the logical implication $\theta\left(t_{2}\right) \leq \theta\left(t_{1}\right) \Rightarrow \theta\left(t_{1}+\lambda\left(t_{2}-t_{1}\right)\right) \leq \theta\left(t_{1}\right), \quad \forall \lambda \in[0,1]$ are equivalent to $f\left(x^{2}\right) \leq f\left(x^{1}\right)$, and $f\left(x^{1}+\lambda\left(x^{2}-x^{1}\right)\right) \leq f\left(x^{1}\right), \forall \lambda \in[0,1]$, respectively.

The next lemma is proved in Cambini and Martein [12] and is given also by Crouzeix [13], without proof. if

Lemma 2. Let $\varphi: I \rightarrow \mathbb{R}$ be differentiable on the interval $I \subset \mathbb{R}$; then $\varphi$ is quasiconvex on $I$ if and only

$$
t_{1}, t_{2} \in I, \varphi\left(t_{2}\right) \leq \varphi\left(t_{1}\right) \Rightarrow \varphi^{\prime}\left(t_{1}\right)\left(t_{2}-t_{1}\right) \leq 0 .
$$

Proof. Let $t_{1}, t_{2} \in I$ such that $t_{1}<t_{2}\left(t_{1}>t_{2}\right)$ and $\varphi\left(t_{1}\right) \geq \varphi\left(t_{2}\right)$. The quasiconvexity of $\varphi$ implies $\varphi(t) \leq$ $\varphi\left(t_{1}\right), \quad \forall t \in\left[t_{1}, t_{2}\right] \quad\left(\forall t \in\left[t_{2}, t_{1}\right]\right)$ so that $\varphi$ is locally non-increasing (locally non-decreasing) at $t_{1}$ and consequently (2) holds. Assume now that (2) holds. If $\varphi$ is not quasiconvex, there exist $t_{1}, t_{2} \in I$ with $t_{1}<t_{2}$ such that $M=\max \left\{\varphi(t), t \in\left[t_{1}, t_{2}\right]\right\}>\max \left\{\varphi\left(t_{1}\right), \varphi\left(t_{2}\right)\right\}$. Let $\bar{t}=\inf \left\{t \in\left[t_{1}, t_{2}\right]: \varphi(t)=M\right\}$; the continuity of $\varphi$ implies the existence of $\varepsilon>0$ such that $\varphi(t)<M, \forall t \in(\bar{t}-\varepsilon, \bar{t})$ and $\varphi(t)>\max \left\{\varphi\left(t_{1}\right), \varphi\left(t_{2}\right)\right\}$, $\forall t \in(\bar{t}-\varepsilon, \bar{t})$. The mean value theorem applied to the interval $[\bar{t}-\varepsilon, \bar{t}]$ implies the existence of $t^{*} \in(\bar{t}-\varepsilon, \bar{t})$ such that $\varphi^{\prime}\left(t^{*}\right)>0$. Consequently, we have $\varphi\left(t^{*}\right)>\varphi\left(t_{2}\right)$ with $\varphi^{\prime}\left(t^{*}\right)>0$ and this contradicts (2).

\section{Proof of Theorem 1.}

It is sufficient to note that if $f$ is differentiable on the open and convex set $X \subset \mathbb{R}^{n}$, then we have, with $\theta(t)=f(x+t y)$,

$$
\theta^{\prime}(t)=\nabla f(x+t y) y
$$

Therefore, on the ground of Lemmas 1 and 2, if we put $x^{1}=x+t_{1} y, \quad x^{2}=x+t_{2} y$, we have $f\left(x+t_{2} y\right) \leq f\left(x+t_{1} y\right) \Rightarrow \nabla f\left(x+t_{1} y\right) y\left(t_{2}-t_{1}\right) \leq 0, \quad$ i.e. $\quad x^{1}, x^{2} \in X, \quad f\left(x^{2}\right) \leq f\left(x^{1}\right) \Rightarrow \nabla f\left(x^{1}\right)\left(x^{2}-x^{1}\right) \leq 0$.

Finally, we point out that the proof of Ponstein [10] can be shortened as follows:

1) Let $f$ be quasiconvex (and differentiable) on the open and convex set $X \subset \mathbb{R}^{n}$, i.e. let

$$
x^{1}, x^{2} \in X, f\left(x^{2}\right) \leq f\left(x^{1}\right) \Rightarrow f\left(\lambda x^{1}+(1-\lambda) x^{2}\right) \leq f\left(x^{1}\right), \forall \lambda \in[0,1] .
$$

By the mean value theorem there exists a number $\xi \in(0,1)$ such that

$$
\nabla f\left(x^{1}+\xi \lambda\left(x^{2}-x^{1}\right)\right) \lambda\left(x^{2}-x^{1}\right)=f\left(x^{1}+\lambda\left(x^{2}-x^{1}\right)\right)-f\left(x^{1}\right) \leq 0 .
$$

Dividing by $\lambda(>0)$ and letting $\lambda \rightarrow 0$, we have $\nabla f\left(x^{1}\right)\left(x^{2}-x^{1}\right) \leq 0$. 
2) Assume conversely that (1) holds and that for $x^{1}, x^{2} \in X$, with $f\left(x^{2}\right) \leq f\left(x^{1}\right)$ there exists a point $x^{3}$ between $x^{1}$ and $x^{2}$ with $f\left(x^{3}\right)>f\left(x^{1}\right)$. Then there exists near $x^{3}$ (e.g. between $x^{1}$ and $x^{3}$ ) also a point $x^{4}$ with $f\left(x^{4}\right)>f\left(x^{1}\right)$ and $\nabla f\left(x^{4}\right)\left(x^{2}-x^{4}\right)>0$. Indeed, if for all $x$ between $x^{1}$ and $x^{3}$ it would hold $\nabla f(x)\left(x^{2}-x\right) \leq 0$ or $f(x) \leq f\left(x^{1}\right)$, then we would have $f\left(x^{3}\right) \leq f\left(x^{1}\right)$ but not $f\left(x^{3}\right)>f\left(x^{1}\right)$. But, be- ing $f\left(x^{2}\right)<f\left(x^{4}\right)$, this implies $\nabla f\left(x^{4}\right)\left(x^{2}-x^{4}\right) \leq 0$, in contradiction with the inequality previously obtained.

\section{References}

[1] De Finetti, B. (1949) Sulle Stratificazioni Convesse. Annali di Matematica Pura ed Applicata, 30, 173-183. http://dx.doi.org/10.1007/BF02415006

[2] Fenchel, W. (1953) Convex Cones, Sets and Functions. Lecture Notes, Princeton University, Princeton.

[3] Arrow, K.J. and Enthoven, A.C. (1961) Quasi-Concave Programming. Econometrica, 29, 779-800. http://dx.doi.org/10.2307/1911819

[4] Avriel, M. (1976) Nonlinear Programming: Analysis and Methods. Prentice-Hall, Englewood Cliffs.

[5] Avriel, M., Diewert, W.E., Schaible, S. and Zang, I. (1988) Generalized Concavity. Plenum Press, New York. http://dx.doi.org/10.1007/978-1-4684-7600-2

[6] Bazaraa, M.S. and Shetty, C.M. (1976) Foundations of Optimization. Springer Verlag, Berlin. http://dx.doi.org/10.1007/978-3-642-48294-6

[7] Bazaraa, M.S., Sherali, H.D. and Shetty, C.M. (1993) Nonlinear Programming. John Wiley \& Sons, New York.

[8] Kemp, M.C. and Kimura, Y. (1978) Introduction to Mathematical Economics. Springer Verlag, New York. http://dx.doi.org/10.1007/978-1-4612-6278-7

[9] Mangasarian, O.L. (1969) Nonlinear Programming. McGraw-Hill, New York.

[10] Ponstein, J. (1967) Seven Kinds of Convexity. SIAM Review, 9, 115-119. http://dx.doi.org/10.1137/1009007

[11] Simon, C.P. and Blume, L. (1994) Mathematics for Economists. W. W. Norton \& Co., New York.

[12] Cambini, A. and Martein, L. (2009) Generalized Convexity and Optimization. Springer, Berlin.

[13] Crouzeix, J.-P. (2005) Criteria for Generalized Convexity and Generalized Monotonicity in the Differentiable Case. In: Hadjisavvas, N., Komlosi, S. and Schaible, S., Eds., Handbook of Generalized Convexity and Generalized Monotonicity, Springer, New York, 89-119. http://dx.doi.org/10.1007/0-387-23393-8_2 